\title{
AÇÃO PIANÍSTICA E COORDENAÇÃO MOTORA: RELAÇÕES INTERDISCIPLINARES ${ }^{1}$
}

\author{
Maria Bernardete Castelan Póvoas², Daniel Silva³, Vânia Eger Pontes ${ }^{4}$ \\ Palavras-chave: ação pianística - coordenação motora - interdisciplinaridade - \\ técnica pianística
}

\begin{abstract}
Resumo:
Este artigo representa parte da pesquisa "ação pianística e coordenação motora - relações interdisciplinares" cuja origem está no fato de o movimento, um ato motor, possibilitar a realização músico-instrumental. As bases teórico-metodológicas são interdisciplinares, com aportes na literatura sobre técnica pianística e demais áreas que tratam do movimento humano. O objetivo estabelecido para esta parte da pesquisa foi levantar e discutir argumentos interáreas, estabelecendo-se conexões teórico-práticas entre aspectos relacionados à coordenação e a atividade pianística.
\end{abstract}

\section{INTRODUÇÃO}

O fato de o elemento meio de uma realização músico-instrumental ser o movimento, um ato motor, é a origem desta proposta de investigação. Levantar pressupostos interdisciplinares com vistas à otimização do desempenho técnico-instrumental com base na investigação sobre o fator do desempenho coordenação motora e o estabelecer conexões teórico-práticas com a ação pianística são objetivos desta pesquisa.

Para este artigo, optou-se pelo destaque a argumentos que definem e explicam fenômenos da coordenação e controle motor que mais diretamente se aplicam à ação pianística e que podem interferir na sua prática. Paralelamente, buscou-se mostrar que movimentos complexos, sobretudo aqueles que são necessários à execução de situações musicais que agregam dois ou mais eventos musicais, dispostos a uma média ou longa distância uns dos outros, podem ser simplificados em sua concepção inicial, por meio da redução das distâncias entre eventos quando da organização do movimento em ciclos (Póvoas, 1999). A citada redução permite que, para grande parte das situações de desempenho músico-instrumental, encontremos a trajetória demovimento mais funcional.

Ao final desta pesquisa, pretende-se avaliar se o conhecimento e o treinamento pianístico conforme a proposta, permitem que a realização de determinadas situações técnicointerpretativas sejam otimizadas tanto nos aspectos técnicos quanto musicais, por meio da

\footnotetext{
${ }^{1}$ Projeto de Pesquisa CEART-UDESC.

${ }^{2}$ Orientadora, Professora do Departamento de Música - Centro de Artes - Florianópolis - SC.

${ }^{3}$ Acadêmico do Curso de Bacharelado em Piano - CEART/UDESC, bolsista de iniciação científica do PROBIC/UDESC.

${ }^{4}$ Acadêmica do Curso de Bacharelado em Piano - CEART/UDESC, bolsista de iniciação científica do $\mathrm{PIBIC/CNPq}$.
}

DAPesquisa, Florianópolis, v.2, n.4, p. 480-490, 2007. 
realização de análises quantitativa (experimento biomecânico utilizando-se a cinemetria ou cinematografia como método de análise) e qualitativa. O experimento será realizado no Laboratório de Biomecânica do Centro de Educação Física, CEFID-UDESC e deverá contar com o auxílio de técnicos e a participação de dois grupos de sujeitos pianistas.

\section{FUNDAMENTAÇÃO TEÓRICA}

Dentre os pressupostos que deram origem à proposta destaca-se aquele de Rasch quando diz que o desempenho humano é "a expressão de vários componentes denominados fatores do desempenho”. (RASCH, 1997: p.183). Esta afirmação motivou-nos a seguir no aprofundamento das relações entre aspectos pertinentes à coordenação motora e questões técnico-instrumentais.

A coordenação, na atividade humana "é a harmonização de todos os processos parciais do ato motor em vista do objetivo, da meta a ser alcançada pela execução do movimento". (MEINEL, 1987: p.2). Coordenação significa "ordenar junto" e o significado desta ordenação se altera dependendo da atividade a que se aplica. Este fator está presente em todas as ações motoras, como na prática pianística, e influi na agilidade. (Moreira, 2000). O corpo humano realiza uma série de movimentos, que emergem de "uma integração entre o indivíduo, a tarefa e o ambiente". (HUMWAY-COOK \& WOOLLACOTT, 2003: p.02).

Quanto maior for o controle e a "perfeição" com que as ações motoras são treinadas e assimiladas, ao nível de domínio ou "integração interna", maiores serão as possibilidade de que se tornem independentes do ambiente (Kaplan, 1987; Knapp, 1989; Rasch, 1997). Não há dúvidas de que a habilidade motora é parte integrante da prática pianística, pois que esta depende de movimentos voluntários para atingir sua meta, a realização sonora. Para esta prática utiliza-se, sobretudo, da habilidade motora fina que requer o controle de pequenos músculos das mãos e dedos, e um alto grau de precisão para tocar as teclas certas, na sequiência e tempo corretos.

Deve-se considerar que exercícios que levam as articulações além do seu arco de movimento podem causar lesão ao comprimir uma base óssea sobre outra, além de provocarem tensão muscular e estiramento nos tendões (Sakai, 2006), muito embora a flexibilidade seja um fator preponderante na ação pianística. Fink (1997) enfatiza a necessidade de uma atenção especial sobre o que denomina de "movimentos fundamentais" 
durante a prática quando se refere à flexibilidade. Estes seriam os movimentos relacionados ao trabalho específico para os dedos, punho, de rotação do braço, entre outros.

A coordenação motora engloba diferentes formas de manifestações, independentes entre si, e tem uma influência preponderante na agilidade (Moreira, 2000) e envolve a ação de várias articulações e pequenos movimentos que se manifestam simultaneamente com o movimento básico. Todo o corpo pode ser subdividido em segmentos que são unidades de coordenação, definida por Santos (2002) como um segmento corporal constituído por dois elementos rotatórios capazes de girarem simultaneamente em sentidos opostos.

São qualidades de proficiência da habilidade motora: a certeza em realizar, reduzir o tempo de um movimento e de minimizar o gasto de energia, o que torna a qualidade do movimento determinante para o seu sucesso (Schmidt \& Craig, 2001). Atos voluntários transformados em automatismos são reflexos de hábitos adquiridos, produto final da aprendizagem motora. Para Kaplan (1987: p.45), “do ponto de vista da execução instrumental, a aquisição e posterior reorganização dos hábitos" estão na base da construção da técnica.

Torna-se essencial que a preparação dos saltos seja acompanhada da sensação corporal durante a execução do movimento utilizado, aliada ao controle cinestésico, condição para a realização de movimentos mais ágeis e no andamento adequado com maior facilidade, por exemplo, além de possibilitar uma consciência da relação entre o movimento e a obtenção de uma determinada sonoridade. Independente dos afastamentos entre segmentos (direito e esquerdo) há situações em que, para desenvolver-se uma melhor consciência das diferenças entre os movimentos essenciais para a execução de diferentes linhas musicais ao mesmo tempo, em suas particularidades de articulação, fraseado, agógica e planos sonoros, faz-se igualmente necessária uma prática minuciosa e organizada do movimento, com treinamento dos segmentos direito e esquerdo separadamente.

Hábitos motores corretos, a partir da individualização de movimentos primários (Finck, 1997) para depois reorganizá-los, permitem ao pianista direcionar sua atenção aos movimentos segmentados e ao aprendizado do conteúdo musical específico para cada mão, constituindo-se em habilidades essenciais à execução de movimentos complexos. Este tipo de treinamento é eficaz e simplifica os conceitos intelectuais e a coordenação motora (Knapp, 1989; Maggil, 2000). Desta forma, a possibilidade de simplificar movimentos em sua concepção inicial, reduzindo-os, teria suporte nestes autores.

Quanto à orientação espacial de movimentos relacionada ao planejamento de distâncias é apontada como uma das estratégias mais importantes a ser utilizada durante o treinamento pianístico. Kochevitsky (1967: p.62) refere-se à realização ao piano de 
“distâncias" entre eventos musicais para as duas mãos como a questão mais difícil a ser resolvida, isto devido à conformação assimétrica do teclado. As mudanças de posições devem então ser previstas e mentalmente preparadas anteriormente à realização do movimento e, quando for o caso, em direção ao evento seguinte, o que exige do pianista um planejamento do trabalho. Assim, no treinamento pianístico de um trecho musical em que as linhas a serem executadas são opostas e distantes entre si, muitas vezes, pelo fato de o executante não conseguir visualizar as duas mãos ao mesmo tempo devido à distância entre eventos, há necessidade de buscar movimentos mais objetivos e mais econômicos para realizá-las.

Segundo Magill, "depois de praticar cada parte independentemente, o aluno pode reuní-las para praticá-las em uma única unidade, com a sua atenção agora dirigida para as solicitações da coordenação temporal e espacial da ação dos braços (...)”. (MAGILL, 2000: p. 279). É "provável que o treino com elementos isolados se tenha revelado mais eficaz, não só porque simplificava os conceitos intelectuais, como também simplificava a coordenação motora” (CROSS apud Knapp, 1989, p.75). "[Quanto] maior for a perfeição com que é aprendido, mais a seqüência dos atos se torna independente do ambiente e mais a integração é interna" (KNAPP, 1989, p.154). Partindo-se dos pressupostos anteriores, o instrumentista deverá, primeiramente, adquirir uma forma motora ou movimento o mais próximo possível daquele que será teoricamente o mais adequado para, posteriormente, dedicar-se ao trabalho daquela forma a fim de torná-la um hábito motor.

Para Kaplan (1987), “dissociação muscular” é o domínio das sensações de contração e de relaxamento e que além de um controle sobre as sensações, a dissociação possibilita desenvolver a capacidade de auto-observação e, igualmente, a controlar e a coordenar conscientemente o próprio corpo em função do objetivo musical a ser atingido.

Quando da escolha e utilização de técnicas e estratégias de treinamento para a execução instrumental, deve-se considerar a coordenação de movimentos discretos e complexos, caso contrário, corre-se o risco de adquirir hábitos insalubres que refletem na qualidade da técnico-sonora, entre outras particularidades referentes à saúde corporal do pianista que variam desde a ineficiência no desempenho, fadiga e mesmo danos fisiológicos “senão irreversíveis, de difícil recuperação". (LEHMKUL \& SMITH, 1997: p. 58).

O aprendizado do piano, da leitura e a formação de uma concepção musical baseada no conhecimento da teoria, desde o seu início, não prescindem do trabalho consciente dos movimentos necessários a cada nova situação de execução. O controle do movimento, quando adequado ao design da obra em estudo, abre a possibilidade de que sejam aplicadas diretamente ou ajustadas a experiências subseqüentes. O papel dos professores de instrumento 
na busca da excelência dos processos de aprendizagem motora ao nível de estratégias de ensino é fundamental. Torna-se essencial conduzir o aluno no sentido de uma vivência e conscientização ótimas, para que os reflexos ao nível de segurança sejam satisfatórios. Da organização do trabalho para uma prática objetiva depende o alcance de metas.

Questões relacionadas ao feedback, informações sensoriais fornecidas ao indivíduo, devem também ser consideradas durante ou após a execução de uma ação. Denominam-se feedback intrínseco (ou proprioceptivo) e feedback extrínseco (ou aumentado). O primeiro é fornecido por meio dos órgãos sensoriais (como a audição e a visão) do próprio sujeito que realizou o movimento e pode fornecer um grande número de informações sobre o movimento realizado, como nível de precisão, postura do corpo e dos membros envolvidos na ação, entre outras informações. Pode auxiliar o músico na detecção e correção de erros cometidos durante ou após a conclusão do movimento durante a execução de uma peça; torna-se mais efetivo em ações que envolvam movimentos lentos e voluntários, pois assim o executante pode fazer compensações durante a ação. $\mathrm{O}$ mesmo não acontece em movimentos rápidos devido ao tempo de resposta não acompanhar o tempo de execução do movimento. (Piekarzievcz, 2004). Quando a ação planejada não alcança sua meta, esta pode estar sendo prejudicada por algum erro no planejamento do movimento, tendo o feedback nesse caso uma função de detectar quais aspectos estão influenciando nesse desempenho.

O feedback extrínseco ou aumentado é fornecido ao indivíduo que executou o movimento a fim de informar-lhe a respeito de suas ações e tem um papel importante no transcorrer do processo de aquisição de uma habilidade motora. Pode ser fornecido pelo professor sobre um movimento executado ou através de uma gravação sonora ou áudio-visual do desempenho. (Maggil, 2000). O professor pode selecionar e enfatizar, primeiramente, o padrão de movimento mais importante envolvido na ação e, depois que o aluno tenha dominado esse padrão, progressivamente fornecer feedback sobre outros aspectos do movimento. (Schmidt e Wrisberg, 2001). O feedback auditivo referente aos parâmetros musicais, têm um importante papel no processo de memorização, influenciando na leitura musical e no desempenho das peças estudadas. (Finney e Palmer, 2003).

O feedback quando dado após a execução de uma ação correta, é chamado de feedback de reforço. Tem a função de assegurar uma maior confiança na ação desempenhada e aumentar a probabilidade que esta ação será repetida em outra situação semelhante. Chiviacowsky e Tani (1997) apontam a importância de desenvolver-se o chamado reforço subjetivo, em que o executante desenvolve a sensibilidade de detecção e correção de seus próprios erros. 


\section{PROCEDIMENTOS METODOLÓGICOS}

Entre as ações realizadas para este trabalho estão: a revisão de literatura articulada em dois eixos, o primeiro percorre abordagens técnico-teóricas da área pianística; o segundo eixo se refere aos pressupostos interdisciplinares de áreas que tratam do movimento humano e que nos permitem estabelecer conexões entre a ação pianística e a coordenação motora, com o objetivo de justificar a proposta de que movimentos complexos podem, em sua concepção inicial, ser reduzidos e, por conseqüência, simplificados. A proposta de simplificar movimentos complexos, por meio da redução de distâncias, foi inicialmente avaliada aplicando-se o recurso à nossa prática e à orientação de alunos em situações musicais específicas. As opções de organização são construídas a partir do texto musical e, posteriormente, aplicadas, observadas e avaliadas quanto a sua operacionalização na prática.

A Figura 1 ilustra um dos trechos escolhidos onde foi aplicado o recurso de redução das distâncias entre eventos visando otimizar o desempenho a partir da orientação da trajetória do movimento a ser seguida. O movimento deve seguir no sentido parabólico e paralelo para os segmentos direito e esquerdo (braços e mãos). No compasso [15], por exemplo, cada ciclo de movimento pode iniciar "tocando-se" as oitavas acentuadas (>) com um gesto para baixo e para a esquerda que serve de impulso para atingir os acordes (m.d., m.e.) em stacatto (.), seguindo-se um movimento para cima e para a direita, em arco (discreto), a partir do próprio acorde que, por sua vez, serve de impulsão para atingir a oitava seguinte ou um novo ciclo. $\mathrm{O}$ desempenho é otimizado se os movimentos forem executados de forma contínua, evitando-se um excessivo movimento do punho para baixo na execução dos acordes que devem ser tocados na continuidade do movimento iniciado nas oitavas. O procedimento contrário causa um maior dispêndio de energia: aumenta a trajetória e diminui a velocidade do movimento.

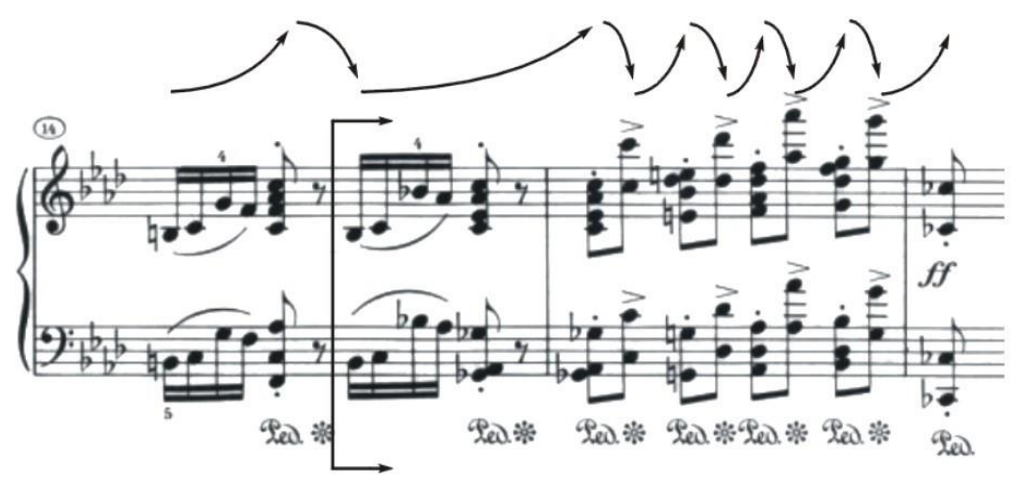

Figura 1a: Prelúdio 18 (compassos [14]-[16]).

Fonte: CHOPIN, 1996: p.37. 
Em um primeiro momento pode-se experimentar os ciclos tocando-se as oitavas e acordes em uma mesma altura, ou suprimir a oitava superior para entender mais facilmente as relações harmônicas por meio da simplificação do movimento através da redução da distância para, somente depois, executar os eventos na altura em que estão escritos.

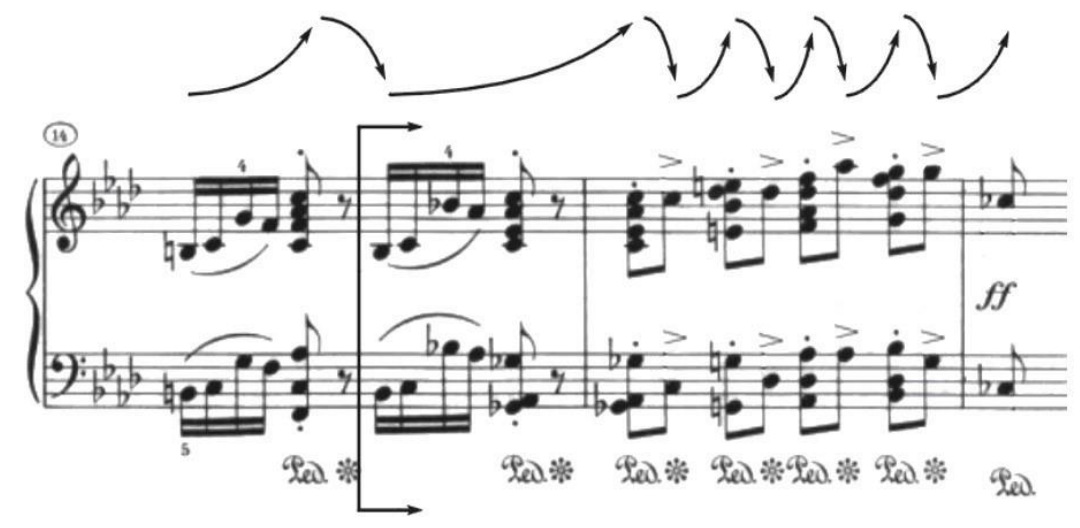

Figura 1b: Redução do movimento - supressão de oitavas. Oitavas suprimidas. Prelúdio 18 (compassos [14][16]). Fonte: CHOPIN, 1996: p.37.

Para a realização do trecho musical anterior, o movimento proposto segundo os ciclos de movimento e os objetivos desta pesquisa, consta de movimentos no sentido parabólico e no sentido horário. Neste modelo, a orientação serve para ambas as mãos. Estratégia equivalente àquela utilizada no modelo anterior (Figura 1) foi aplicada à situação técnico-musical mostrada na Figura 2a e 2b (redução), mostrando-se igualmente funcional.

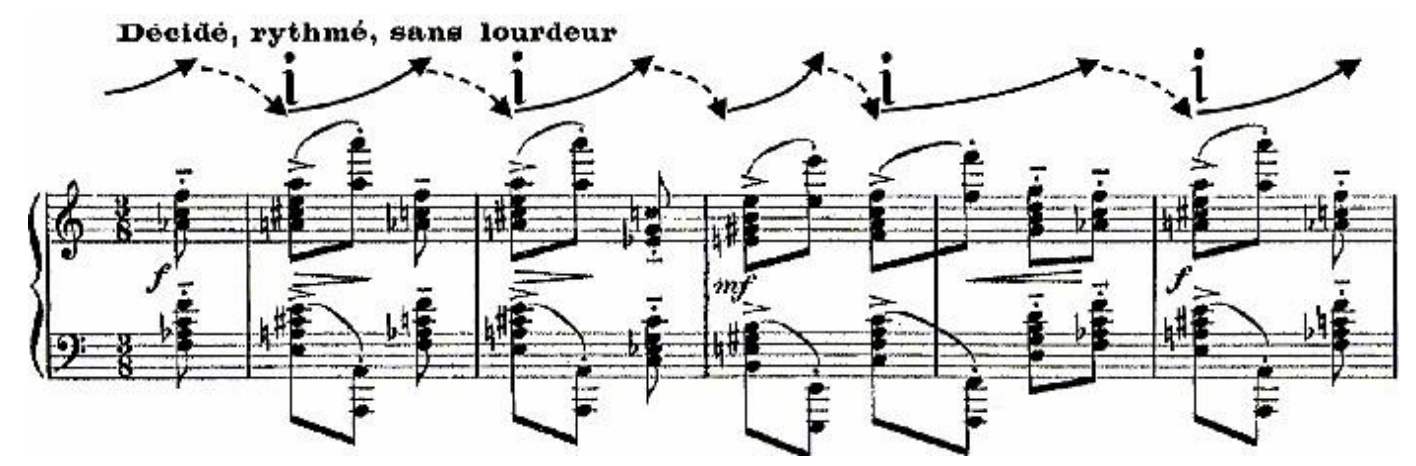

Figura 4a: Étude XII, “Pour Les Accords” (comp. [1]-[3]) de Debussy. Fonte: DEBUSSY (1916, p.26) 


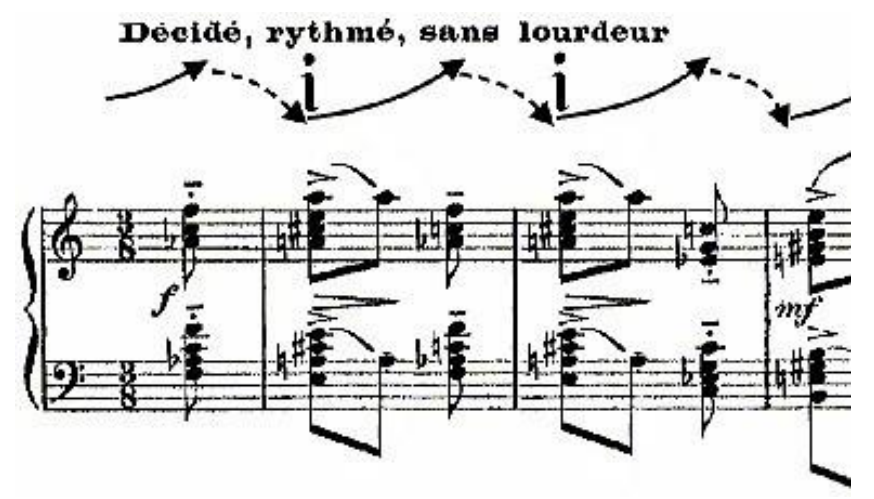

Figura 4a: Redução do movimento - supressão de oitavas. Étude XII, "Pour Les Accords" (comp. [1][3]) de Debussy. Fonte: DEBUSSY (1916, p.26).

\section{DISCUSSÃO E RESULTADOS}

A aplicação dos modelos na prática de alunos apresentou, no decorrer das aulas semanais, uma melhora significativa no resultado técnico e interpretativo de execução. Observou-se uma melhora no nível de atenção ao resultado sonoro. A questão mecânica, uma vez superada pela compreensão das possibilidades e superação dos limites deixou de ser, em muitos casos, o principal foco do treinamento. Os alunos sentiram-se mais seguros e passaram a desenvolver, cada vez mais e com maior prontidão, a capacidade de encontrar as melhores soluções mecânicas em função das solicitações de execução da peça em estudo.

Com a prática de redução e simplificação de trechos musicais mais complexos, em termos de densidade da escrita e de sequiência de eventos a serem executados dispostos à média e longa distância entre si, os alunos tiveram oportunidade de pesquisar, experimentar, tentar possibilidades e de comprovar suas próprias opções ou aquelas orientadas pelo professor. Verificou-se, na maior parte das experiências pessoais e com alunos, que a preparação de um ato motor complexo a partir de sua prévia simplificação de movimentos por redução das distâncias, abre a possibilidade de que mais rapidamente se adquira e domine a habilidade motora de executá-los.

A possibilidade de sucesso e de atingir patamares de desempenho além dos habituais, após a comprovação da eficiência do mecanismo adotado a partir da experiência pessoal, tem se mostrado motivadora. Detalhes sobre os "acertos" são discutidos e reafirmados com o objetivo de se estabelecer hábitos motores funcionais permanentes. Dizemos funcionais 
porque quando o foco é encontrar a solução técnica mais adequada para execução de uma situação musical específica e em função da melhor mecânica do movimento para um(a) aluno(a) em particular, as características individuais de cada um devem ser consideradas.

Dependendo do tempo de experiência com o piano e da densidade da escrita é necessário que movimentos discretos (dedos, punho) sejam trabalhados separadamente, ou que a parte destinada ao trabalho dos segmentos direito e esquerdo sejam praticados individualmente. A individualização ou fragmentação de movimentos simplifica os conceitos intelectuais, como também a coordenação motora. Esta estratégia permite ao pianista concentrar sua atenção no movimento dos segmentos separadamente e aprender o conteúdo musical (partitura) determinado para cada mão (ou lado do corpo) sem o compromisso de fixar-se na coordenação de todo o conjunto de eventos musicais ao mesmo tempo. $\mathrm{O}$ aprendizado pode ser otimizado e, conseqüentemente, o tempo de estudo. Posteriormente, as ações podem ser reorganizadas, constituindo-se assim as habilidades motoras necessárias para a execução do trecho musical na sua totalidade. Este tipo de trabalho auxilia no sentido de evitar, desde os primeiros contatos com uma obra, contrações corporais desnecessárias, facilitando o controle e a coordenação dos movimentos.

\section{CONSIDERAÇÕES FINAIS}

Com base nos pressupostos levantados e na aplicação do recurso proposto em situações de estudo e de aula, verificou-se que a preparação de um ato motor complexo a partir de sua prévia simplificação mostra-se eficiente, pois possibilita a aquisição da habilidade motora de executá-lo em espaço de tempo menor do que em situações de execução equivalentes em que o recurso não foi aplicado.

A aplicação dos modelos ( 1 e 2) mostrou que, para a sua execução pianística, faz-se necessária uma prática atenta ao percurso dos movimentos, uma vez que hábitos motores corretos são transferíveis para situações similares ou adaptáveis a situações semelhantes. Deve-se também considerar o fato de que, sobre o trabalho intensivo de habilidades motoras, desenvolvem-se hábitos sobre os quais nosso domínio técnico se fundamenta. Estabelecer-se hábitos motores corretos desde o início do estudo do piano ou mesmo em níveis mais avançados da experiência musical torna-se um procedimento essencial para o desenvolvimento do trabalho técnico-interpretativo e de sua eficiência. 
A evidência de que a prevenção e a solução de problemas que interferem no desempenho instrumental estão, em grande parte, em argumentos de áreas, como fisiologia, ergonomia, biomecânica vem, cada vez mais, justificando a realização de estudos interdisciplinares e de procedimentos experimentais. Métodos de análise biomecânicos possibilitam medições de parâmetros cinemáticos de movimentos executados por pianistas e os resultados, tanto os matemáticos quanto visuais, podem ser essenciais no auxílio e avaliação de diferentes fatores que interagem no desempenho do instrumentista.

A investigação e formulação de recursos técnicos, aplicáveis a situações específicas de execução, traz à tona argumentos que auxiliam no desenvolvimento de estratégias de treinamento músico-instrumental, ao mesmo tempo em que ampliam as possibilidades de melhoria no nível do desempenho pianístico. As informações até então obtidas poderão contribuir como fonte de informação para profissionais e alunos. O diálogo interáreas e as conexões resultantes constituem-se em um campo de investigação ainda em aberto na pesquisa em práticas músico-instrumentais, no Brasil.

\section{REFERÊNCIAS BIBLIOGRÁFICAS}

CHIVIACOWSKY, Suzete e TANI, Go. Efeitos da frequiência de conhecimento de resultados na aprendizagem de diferentes programas motores generalizados. Revista Paulista de Educação Física: v.1, n.11, p. 15-26, 1997.

CHOPIN, Frédéric. Préludes. Munich: Henle Verlag, 1996.

DEBUSSY, Claude A. Étude XII “Pour Les Accords". Paris: Durand, 1978, p.10.

FINNEY, Steven A. \& PALMER, Caroline. Auditory feedback and memory for music performance: Sound evidence for an encoding effect. Memory \& Cognition: v.1, n.31, p. 5164, 2003.

FINK, Seymour. Mastering Piano Technique. A Guide For Students, Teachers, And Performance. Oregon: Amadeus Press, 1997.

GINASTERA, Alberto. Sonata n.1. New York: Boosey \& Hawkes, 1990.

KAPLAN, José Alberto. Teoria da Aprendizagem Pianística. Porto Alegre: Movimento, 1987.

KNAPP, Bárbara. Desporto e Motricidade. São Paulo: Compendium, 1989.

KOCHEVITSKY, G. (1967). The Art of Piano Playing: A Cientific Approach. New York: Summy-Birchard.

LEHMKUL, L. Don; SMITH, Laura K. Cinesiologia Clínica de Brunnstron. 5. ed. São Paulo: Manole, 1997.

MAGILL, Richard A. Aprendizagem motora - conceitos e aplicações. Tradução de Aracy

Mendes da Costa, $2^{\mathrm{a}}$ reimpressão. São Paulo: Edgard Blücher, 2000.

MEINEL, Curt. Motricidade I: Teoria da Motricidade Esportiva sob o Aspecto

Pedagógico. Tradução de Sonnhilde von der Heide. São Paulo: Ao Livro Técnico, 1987.

MOREIRA, Miguel. A coordenação. Ludens, Ciências do Desporto: V.16, n.4, Out. 2000. 
NIGG, B.M., HERZOG W. (1994) Biomechanics of The Musclo-Skeletal System. Toronto: Wiley\&Sons.

PÓVOAS, Maria Bernardete Castelan. Princípio da Relação e Regulação do ImpulsoMovimento. Possíveis Reflexos na Ação Pianística. Tese de Doutorado, 1999, Universidade Federal do Rio Grande do Sul.

RASCH, Philip J. Cinesiologia e Anatomia Aplicada. Tradução de Marcio Moacyr de Vasconcelos. Rio de Janeiro: Guanabara Koogan, 1997.

SAKAI, Naotaka et al. Hand Span and Digital Motion on the Keyboard: Concerns of Overuse Syndrome in Musicians. The Journal of Hand Surgery: n.5, p. 830-835, 2006.

SANTOS, Ângela. Biomecânica da Coordenação Motora. $2^{\mathrm{a}}$ ed. São Paulo: Summus, 2002. SCHMIDT, Richard; WRISBERG, Craig. Aprendizagem e Performance Motora: Uma abordagem da aprendizagem baseada no problema. Tradução de Ricardo Demétrio de Souza Peterson et al. Porto Alegre: Artmed, 2001.

PIEKARZIEVCZ, Luiz E. Efeitos do feedback extrínseco aumentado no processo de aprendizagem de uma habilidade motora fechada. Dissertação de Mestrado, 2004, Universidade Federal do Paraná.

SHUMWAY-COOK, Anne; WOOLLACOTT, Marjorie H. Controle motor: teoria e aplicações práticas. 2. ed. Barueri: Manole, 2003. 